\title{
Low-Dimensional Nanomaterials and Their Functional Architectures: Synthesis, Properties, and Applications
}

\author{
Weilu Gao, ${ }^{1}$ Ciyuan Qiu, ${ }^{2}$ and Xiaowei $\mathrm{He}^{3}$ \\ ${ }^{1}$ Department of Electrical and Computer Engineering, Rice University, Houston, TX 77005, USA \\ ${ }^{2}$ Department of Electrical Engineering, Shanghai Jiao Tong University, Shanghai 200240, China \\ ${ }^{3}$ Los Alamos National Laboratory, Los Alamos, NM 87545, USA \\ Correspondence should be addressed to Weilu Gao; weilu.gao@rice.edu
}

Received 10 September 2017; Accepted 14 September 2017; Published 26 October 2017

Copyright (C) 2017 Weilu Gao et al. This is an open access article distributed under the Creative Commons Attribution License, which permits unrestricted use, distribution, and reproduction in any medium, provided the original work is properly cited.

The objective of this special issue is to address recent research trends and developments in the synthesis and applications of low-dimensional materials and their functional architectures. A substantial number of papers were submitted, and after a thorough peer review process, eight papers were selected to be included in this special issue. These papers cover advances in diverse topics, covering a wide range of lowdimensional materials. We believe that the original papers collected in this special issue highlight the contemporary topics in research related to low-dimensional materials and will introduce readers to the latest advances in this field.

In the paper by J. C. Briones et al., $\mathrm{SnO}_{2}$ nanowires were first grown using the horizontal vapor phase growth (HVPG) technique. Next, the authors found that the length of nanowires was significantly enhanced by the application of EMF. The aspect ratio, as well as the density of the fabricated nanowires, increased with increasing magnetic field intensity.

In the paper by H. N. T. Phung et al., $\mathrm{MoS}_{2} / \mathrm{TiO}_{2}$ heterostructure thin films were fabricated using sol-gel and chemical bath deposition methods. Crystal structure, surface morphology, chemical states of all elements, and optical property of the obtained thin films were fully characterized by various techniques. The obtained $\mathrm{MoS}_{2} / \mathrm{TiO}_{2}$ heterostructure thin films exhibit higher visible light photocatalytic activity than that of pure $\mathrm{MoS}_{2}$ and $\mathrm{TiO}_{2}$ counterparts. The optimization of $\mathrm{MoS}_{2} / \mathrm{TiO}_{2}$ fabrication process together with the mechanism of the enhancement was also discussed.

The paper by B. Fan et al. described the fabrication of spawns structure of rod-like $\mathrm{ZnO}$ wrapped in the cellulose nanofibers through a facile one-step hydrothermal method. The composite aerogel in a wax matrix exhibited excellent electromagnetic wave absorption performance over 2-18 GHz.

The paper by X. Lu et al. developed a glancing angle deposition (GLAD) technique to generate $\mathrm{SiO}_{2}$ nanorods for a low-cost superhydrophobic functional nanostructured surface. The wettability of the surface was characterized under various fabrication parameters, and a maximum contact angle of $157^{\circ}$ was obtained. Furthermore, optically transparent films have been demonstrated to serve as superhydrophobic functional nanostructures for optical applications.

The paper by W. Liu et al. investigated the influence of heat treatment on the morphologies of copper nanoparticles based films on glass slides with a spin coating method. Using TG, XRD, and SEM techniques, it showed that heat treatment can modify the sizes and morphologies of copper nanoparticles. Significant coarsening of copper nanoparticles occurs when the heating temperature is above $200^{\circ} \mathrm{C}$.

The paper by $\mathrm{W}$. Hu and X. Yuan reported a facile route to synthesize $\mathrm{SnO}_{2}$ nanospheres at large scale. The $\mathrm{SnO}_{2}$ nanospheres were synthesized through a simple and effective hydrothermal method with average diameter of $40 \mathrm{~nm}$. The as-prepared product was used as the photocatalyst for several dyes under UV light irradiation. The results indicate that these nanospheres have high photocatalytic activities and might be a potential candidate in wastewater purification.

The paper by L. Aprilia et al. is about a microcantilever gas sensor based on zinc oxide microrods. $\mathrm{ZnO}$ microrods were grown on the microcantilever surface and the measurement of resonance frequency of the microcantilever vibrations under the exposure of $\mathrm{CO}$ gas was carried out. The results showed that the resonance frequency is shifted by water vapor 
condition. An exchange mechanism of $\mathrm{CO}$ and water was used to explain the shift of resonance frequency.

The paper by $\mathrm{H}$. Huang et al. studied length dependence of carbon nanotubes on the viscosity of carbon nanotubepolymer composites. The effects of nanotube length and weight fraction on the polyisobutylene matrices were evaluated with thermal and rheological testing. It showed that viscosity of the nanocomposite systems depended on the nanotube length, indicating the non-Einstein-like behavior of the viscosity in these composite systems.

\section{Acknowledgments}

We would like to thank all the authors who contributed to this special issue. This publication would not be possible without the participation of our expert reviewers who provided vital constructive feedback and criticism throughout the review process.

Weilu Gao

Ciyuan Qiu

Xiaowei He 

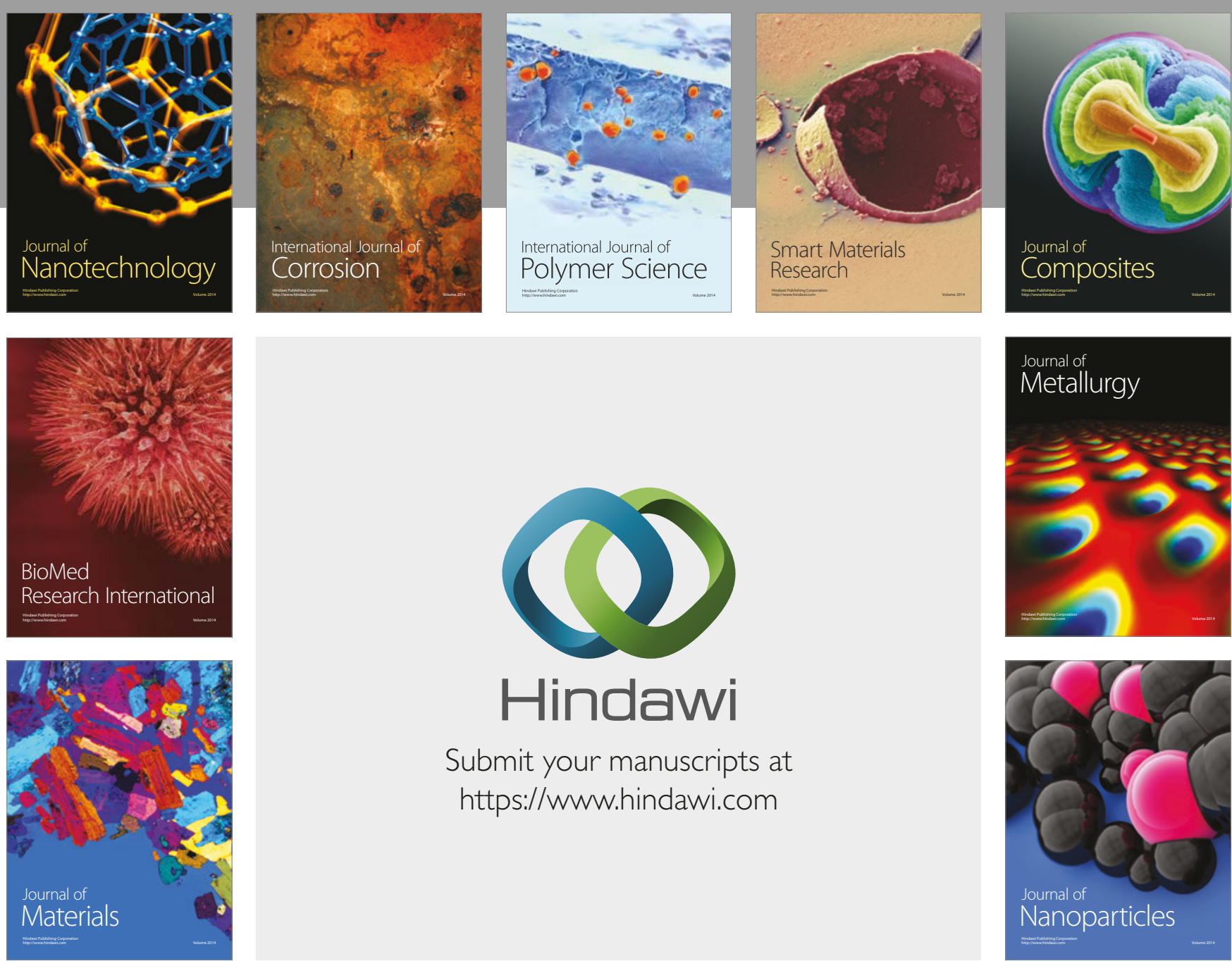

\section{Hindawi}

Submit your manuscripts at

https://www.hindawi.com
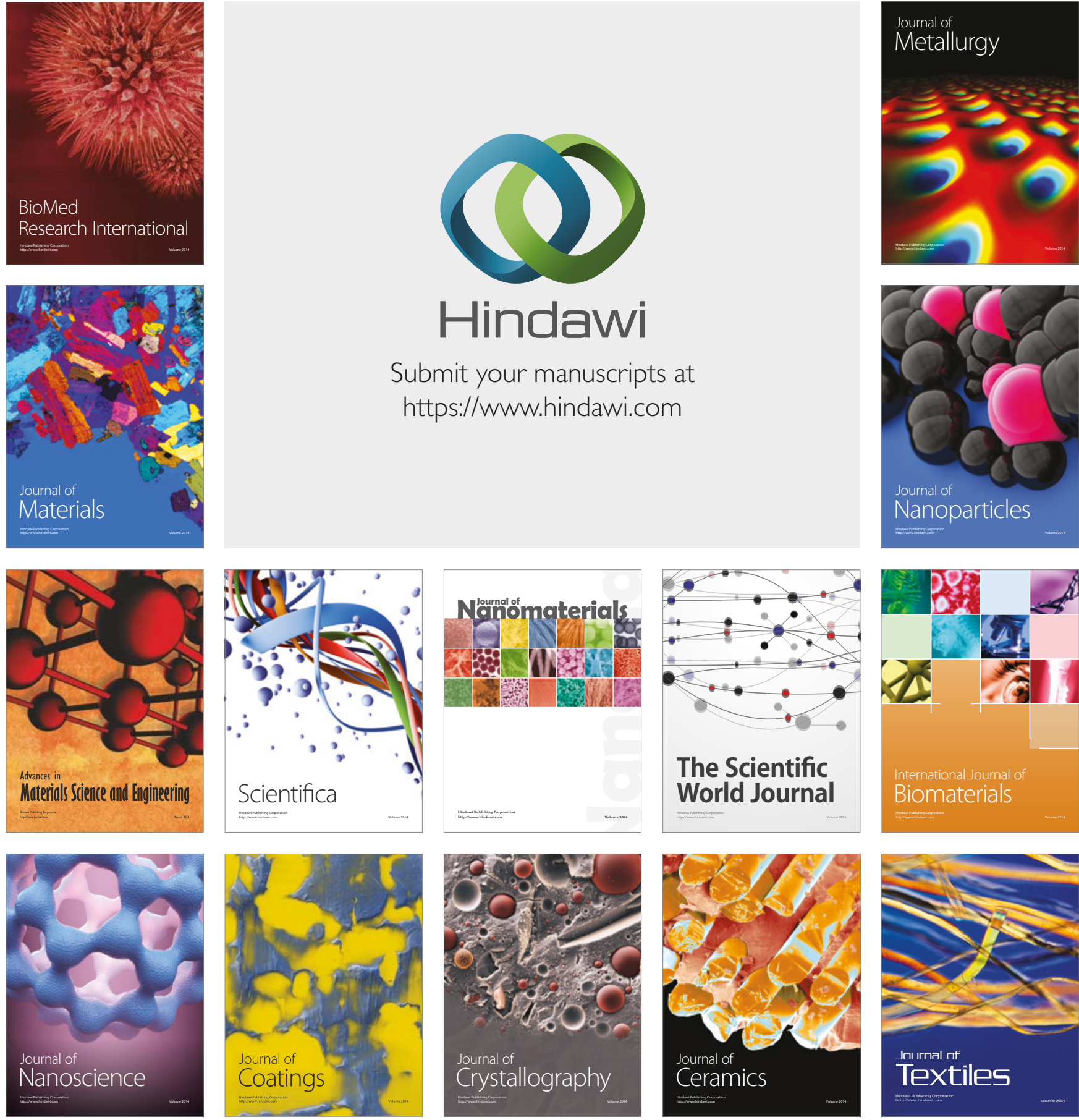

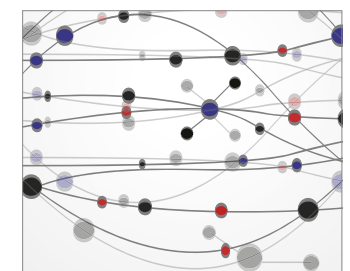

The Scientific World Journal
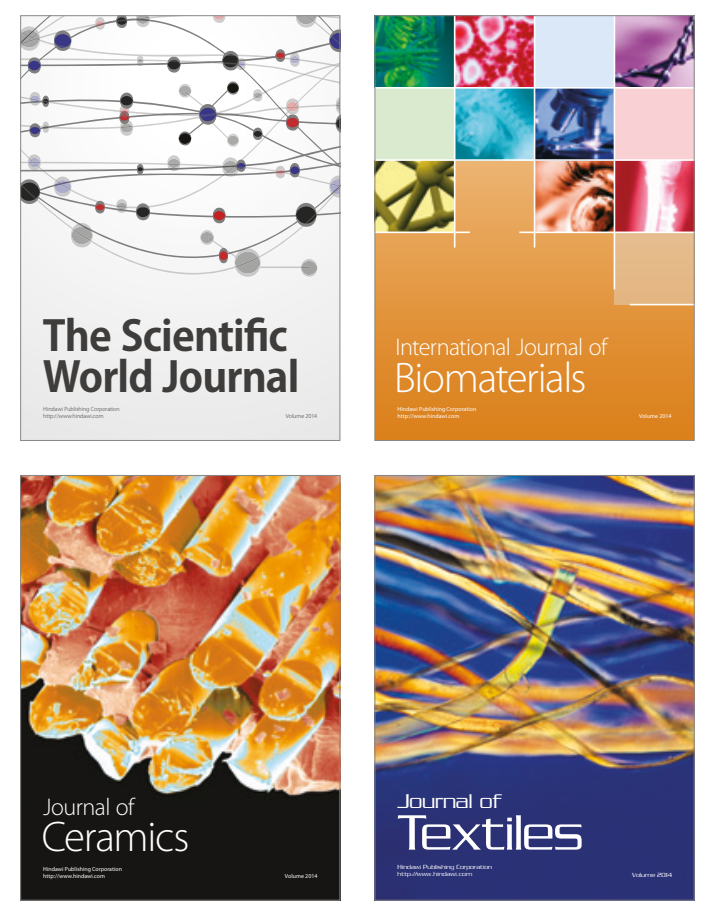